\title{
Epibiontes de Lepidochelys olivacea (Eschscholtz, 1829) (Reptilia: Testudinata: Cheloniidae) en la región centro sur de Chile
}

\author{
Epibionts from Lepidochelys olivacea (Eschscholtz, 1829) (Reptilia: Testudinata: Cheloniidae) \\ in the central south region of Chile
}

Leyla Miranda ${ }^{1}$ y Rodrigo A. Moreno $^{2}$

${ }^{1}$ Instituto de Fomento Pesquero. Casilla 8-V, Valparaiso, Chile.

${ }^{2}$ Departamento de Zoología, Facultad de Ciencias Naturales y Oceanográficas,

Universidad de Concepción. Casilla 160-C, Concepción, Chile.

rodmoren@udec.cl

\begin{abstract}
Data on epibionts found on the carapace, fins and cloaca of five olive-ridley sea turtle Lepidochelys olivacea
\end{abstract}

specimens stranded in the years 2000 and 2001 in the central south region of Chile.

Key words: Epibionts, Lepidochelys olivacea, Reptilia
Lepidochelys olivacea (Eschscholtz, 1829), tortuga olivácea, se distribuye por todos los océanos del mundo (Márquez 1990) y constituye, al igual que otras especies de tortugas marinas, substrato para una gran variedad de epibiontes marinos (Dood 1988). Esta nota reporta cinco especies de epibiontes obtenidos de tortugas oliváceas varadas en el litoral de la VIII Región de Chile, durante los años 2000 y 2001. Las tortugas varadas fueron transportadas al Laboratorio de Taxidermia del Departamento de Zoología de la Universidad de Concepción para las respectivas necropsias. Los epibiontes se recolectaron del caparazón, aletas y cloaca y fueron depositados en el Museo de Zoología de la Universidad de Concepción (MZUC) (Tabla 1).

Se obtuvieron dos grupos de epibiontes: crustáceos e hidrozoos. En el primer grupo, tres especies son cirripedios y una es decápodo. De los cirripedios, Lepas anatifera Linné es cosmopolita y común en todos los océanos, Verruca laevigata (Sowerby) es común en Perú y Chile y Balanus laevis Bruguière es común desde California hasta las islas Falkland y sur de Brasil por el Atlántico (Nilsson-Cantell 1957). El decápodo Planes cyaneus Dana es de amplia distribución en el Pacífico central (Retamal 1981).

En relación con la superficie corporal de las tortugas donde se encontraron los epibiontes, cinco tortugas hospedaban en el caparazón colonias de un hidrozoario indeterminado, en cambio, sólo en dos se encontró el cirripedio Lepas anatifera. De los epibiontes de las aletas, sólo dos tortugas hospedaban al cirripedio incrustante Balanus laevis y sólo una a Verruca laevigata. Este último cirripedio perfora las aletas de las tortugas causando heridas. Solamente se registró una tortuga que hospedaba en su zona cloacal al decápodo Planes cyaneus.

Desde el punto de vista ecológico, la principal relación que se establece entre las colonias de hidrozoarios y de los cirripedios Lepas anatifera, Verruca laevigata y Balanus laevis como epibiontes de tortugas marinas, es la utilización de la superficie corporal como substrato de asentamiento y a su vez como medio de dispersión y obtención de alimento (Barnes 1986). Para el caso de Planes cyaneus se establece una interacción comensalista, debido a que este decápodo consume los desechos fecales de la cloaca de la tortuga. Sin embargo, no se conocen mayores detalles de esta interacción.

El conocimiento de la composición taxonómica, de los patrones de distribución y de la abundancia de estas especies epibiontes, representa un elemento importante para entender mejor la relación entre estos hospedadores y sus epibiontes. Estos antecedentes permiten establecer aproximaciones como aquélla propuesta por Eckert \& Eckert (1988), quienes infieren rutas de migración prereproductivas para la tortuga laúd (Dermochelys coriacea) en EE.UU., basada en datos de epibiontes, o aquélla propuesta por Killingley \& Lutcavage (1983), sobre patrones de movimientos para Caretta caretta utilizando datos de balánidos epizoicos. 
Tabla 1

Epibiontes encontrados en cinco ejemplares de Lepidochelys olivacea varados en la zona centro sur de Chile, años 2000 y 2001

Epibionts collected from five Lepidochelys olivacea specimens stranded in the central south region of Chile, years 2000 and 2001

\begin{tabular}{|c|c|c|c|}
\hline Fecha registro tortugas & Localidad & Epibiontes & Región corporal de la tortuga \\
\hline 28-jun-2000 & $\begin{array}{l}\text { Laraquete } \\
\left(37^{\circ} 07^{\prime} \mathrm{S} ; 73^{\circ} 11^{\prime} \mathrm{W}\right)\end{array}$ & $\begin{array}{l}\text { Hydrozoa indeterminado } \\
\text { (MZUC 27028) } \\
\text { Balanus laevis } \\
\text { (MZUC 27033; } 3 \text { ejemplares) }\end{array}$ & $\begin{array}{l}\text { Caparazón } \\
\text { Aletas }\end{array}$ \\
\hline 10-jul-2000 & $\begin{array}{l}\text { Caleta Lenga } \\
\left(36^{\circ} 47^{\prime} \mathrm{S} ; 73^{\circ} 07^{\prime} \mathrm{W}\right)\end{array}$ & $\begin{array}{l}\text { Balanus laevis } \\
\text { (MZUC 27034; } 2 \text { ejemplares) } \\
\text { Hydrozoa indeterminado } \\
\text { (MZUC 27028) }\end{array}$ & $\begin{array}{l}\text { Aletas } \\
\text { Caparazón }\end{array}$ \\
\hline 01-jun-2001 & $\begin{array}{l}\text { Caleta Lenga } \\
\left(36^{\circ} 47^{\prime} \mathrm{S} ; 73^{\circ} 07^{\prime} \mathrm{W}\right)\end{array}$ & $\begin{array}{l}\text { Hydrozoa indeterminado } \\
\text { (MZUC 27028) }\end{array}$ & Caparazón \\
\hline 02-jun-2001 & $\begin{array}{l}\text { Pingueral } \\
\left(36^{\circ} 33^{\prime} \mathrm{S} ; 72^{\circ} 56^{\prime} \mathrm{W}\right)\end{array}$ & $\begin{array}{l}\text { Lepas anatifera } \\
\text { (MZUC 27031; } 1 \text { ejemplar) } \\
\text { Planes cyaneus } \\
\text { (MZUC 27029; } 1 \text { ejemplar) } \\
\text { Hydrozoa indeterminado } \\
\text { (MZUC 27028) }\end{array}$ & $\begin{array}{l}\text { Caparazón } \\
\text { Cloaca } \\
\text { Caparazón }\end{array}$ \\
\hline 04-jul-2001 & $\begin{array}{l}\text { Coronel } \\
\left(37^{\circ} 04^{\prime} \mathrm{S} ; 73^{\circ} 10^{\prime} \mathrm{W}\right)\end{array}$ & $\begin{array}{l}\text { Lepas anatifera } \\
\text { (MZUC 27030; } 1 \text { ejemplar) } \\
\text { Hydrozoa indeterminado } \\
\text { (MZUC 27028) } \\
\text { Verruca laevigata } \\
\text { (MZUC 27032; } 5 \text { ejemplares) }\end{array}$ & $\begin{array}{l}\text { Caparazón } \\
\text { Caparazón } \\
\text { Aletas }\end{array}$ \\
\hline
\end{tabular}

Las especies de epibiontes reportadas en esta nota son comunes en las aguas del Océano Pacífico. Sin embargo, su presencia como tal en Lepidochelys olivacea no había sido documentada para Chile, lo que constituye el primer antecedente sobre el tema.

\section{Agradecimientos}

Especialmente al Profesor Walter Sielfeld K. (Universidad Arturo Prat) por las sugerencias críticas al manuscrito.

Esta nota forma parte de la tesis del primer autor para optar al Título de Biólogo Marino de la Universidad de Concepción.

\section{Literatura Citada}

Barnes R. 1986. Zoología de los Invertebrados, 1157 p. Cuarta Edición. Editorial Interamericana, México.

Dood CK. 1988. Synopsis of the biological data on the loggerhead sea turtle. U.S. Fish and Wildlife Service Biological Report 88: 1-110.
Eckert KL \& SA Eckert. 1988. Pre-reproductive movements of leatherback sea turtles, Dermochelys coriacea, nesting in the Caribbean. Copeia (2): 400-406.

Killingley J \& M Lutcavage. 1983. Loggerhead turtle movements reconstructed from 180 and $13 \mathrm{C}$ profiles from commensal barnacle shells. Estuarine, Coastal and Shelf Science 16: 345-349.

Márquez M. 1990. FAO Species Catalogue. Vol. 11: Sea turtles of the world. An annotated and illustrated catalogue of sea turtle species known to date. FAO Fisheries Synopsis 125 (11):1- 81 .

Nilsson-Cantell C. 1957. Reports of the Lund University Chile Expedition 1948-49, No 31. Lund Universitets Arsskrift. N. F. Avd. 2.53 (9). Kungl. Fysiogr. Sallskap. Handl. 68 (9): 1-25.

Retamal M. 1981. Catálogo ilustrado de los crustáceos decápodos de Chile. Gayana Zoología 44: 1-110. 